Published in final edited form as:

Fam Cancer. 2015 June ; 14(2): 297-306. doi:10.1007/s10689-014-9758-8.

\title{
Mutations of HNRNPAO and WIF1 predispose members of a large family to multiple cancers
}

\author{
Chongjuan Wei, \\ Department of Epidemiology, The University of Texas MD Anderson Cancer Center, Houston, TX \\ 77030, USA
}

\section{Bo Peng,}

Department of Bioinformatics and Computational Biology, The University of Texas MD Anderson Cancer Center, Houston, TX 77030, USA

\section{Younghun Han,}

Department of Epidemiology, The University of Texas MD Anderson Cancer Center, Houston, TX 77030, USA

\section{Wei V. Chen,}

Clinical Applications and Support, The University of Texas MD Anderson Cancer Center, Houston, TX 77030, USA

\section{Joshua Rother,}

Department of Epidemiology, The University of Texas MD Anderson Cancer Center, Houston, TX 77030, USA

\section{Gail E. Tomlinson,}

Department of Pediatrics and Greehey Children's Cancer Research Institute, University of Texas Health Science Center at San Antonio, San Antonio, TX 78229, USA

Hamon Center for Therapeutic Oncology Research, University of Texas Southwestern Medical Center, Dallas, TX 75225, USA

\section{Richard Boland,}

GI Cancer Research Laboratory, Baylor University Medical Center, 250 Hoblitzelle, 3500 Gaston Avenue, Dallas, TX 75246, USA

\section{Marc Chaussabel,}

GI Cancer Research Laboratory, Baylor University Medical Center, 250 Hoblitzelle, 3500 Gaston Avenue, Dallas, TX 75246, USA

Marsha L. Frazier, and

\footnotetext{
Correspondence to: Marsha L. Frazier, mlfrazier@mdanderson . org; Christopher I. Amos, Christopher. I. Amos@Dartmouth. edu.

Chongjuan Wei and Bo Peng have contributed equally to this work.

Conflict of interest The authors declare that they have no conflict of interest.

Electronic supplementary material The online version of this article (doi:10.1007/s10689-014-9758-8) contains supplementary material, which is available to authorized users.
} 
Department of Epidemiology, The University of Texas MD Anderson Cancer Center, Houston, TX 77030, USA

\section{Christopher I. Amos}

Department of Community and Family Medicine, Center for Genomic Medicine, Geisel School of Medicine, Dartmouth College, Lebanon, NH 03766, USA

Marsha L. Frazier: mlfrazier@mdanderson.org; Christopher I. Amos: Christopher.I.Amos@Dartmouth.edu

\section{Abstract}

We studied a large family that presented a strong familial susceptibility to multiple early onset cancers including prostate, breast, colon, and several other uncommon cancers. Through targeted gene, linkage, and whole genome sequencing analyses, we show that the presence of a variant in the regulatory region of $H N R N P A O$ associated with elevated cancer incidence in this family (Hazard ratio $=7.20, p=0.0004$ ). Whole genome sequencing identified a second rare protein changing mutation of WIFI that interacted with the HNRNPAO variant resulting in extremely high risk for cancer in carriers of mutations in both genes $\left(p=1.98 \times 10^{-13}\right)$. Analysis of downstream targets of the mutations in these two genes showed that the HNRNPAO mutation affected expression patterns in the PI3 kinase and ERK/MAPK signaling pathways, while the WIF1 variant influenced expression of genes that play a role in NAD biosynthesis. This is a first report of variation in HNRNPAO influencing common cancers or of a striking interaction between rare variants coexisting in an extended pedigree and jointly affecting cancer risk.

\section{Keywords}

Whole genome sequencing; Expression analysis; Linkage analysis; Complex disease; Colon cancer; Prostate cancer

\section{Introduction}

While extensive family studies have shown that almost all cancers are influenced by genetic factors, rather little of the overall genetic cancer burden has to date been assigned to specific genetic factors. It has been hypothesized that much of the missing heritability explaining common diseases like cancer results from an inadequate assessment of interactions that may jointly substantially affect risk. In this study, we studied a large family presenting initially through a proband with very early onset breast cancer. Subsequently, a second relative in the family developed an extremely early onset colorectal cancer. Further contact with the family showed that a large number of relatives had developed cancers, with many common cancers occurring at an early age. Analysis of mutations in all known cancer predisposing loci failed to yield any relevant findings. We therefore undertook a comprehensive analysis of the family, including linkage analysis using dense single nucleotide polymorphism analysis, whole genome sequencing of key family members and expression analysis to characterize findings associated with specific mutations that were identified. Here we report two novel mutations in heterogeneous nuclear ribonucleoprotein A0 (HNRNPAO) and WNT Inhibitory Factor 1 (WIFl) genes in this family, which are associated with the elevated incidence of cancers in this family. 


\section{Materials and methods}

\section{Patients}

Families were accrued through collaboration among the University of Texas MD Anderson Cancer Center, the Baylor University Medical Center and the University of Texas Southwestern Medical Center. Participants included family members with cancer; firstdegree relatives without cancer and more distant relatives used to reconstruct any deceased or missing family members as well as spouses of family members. All participants were enrolled on Institutional Review Board-approved protocols at all institutions and were asked to provide blood for genetic analysis and expression profiling after signing informed consent forms. Participants also completed a health and diet questionnaire and medical history information was requested from all individuals.

\section{DNA extraction}

Blood from each participant was collected with a Vacutainer tube containing ethylenediamine tetraacetic acid (EDTA) (Becton Dickinson Vacutainer System, Rutherford, NJ, USA) and DNA was isolated with Qiagen Kits (Qiagen Inc., Valencia, CA, USA) according to the manufacturer's instructions.

\section{Expression analyses}

Total RNA was isolated using the RNeasy Mini kit (Qiagen, Valencia, CA, USA) according to manufacturer's instructions. RNA integrity was assessed using an Agilent 2100 Bioanalyzer (Agilent, Palo Alto, CA, USA). Affymetrix GeneChips were used for expression analysis. Target labeling was performed according to the manufacturer's standard protocol (Affymetrix Inc., Santa Clara, CA, USA). Biotinylated cRNA targets were purified and subsequently hybridized to Affymetrix HG-U133A and U133B GeneChips (>44,000 probe sets). Arrays were scanned using an Affymetrix confocal laser scanner. Microarray Suite, Version 5.0 (MAS 5.0; Affymetrix) software was used to assess fluorescent hybridization signals, to normalize signals, and to evaluate signal detection calls. Normalization of signal values per chip was achieved using the MAS 5.0 global method of scaling to the target intensity value of 500 per GeneChip.

\section{Sanger sequencing and mutation analysis}

Sanger sequencing method was used to analyze mutations in known cancer predisposing loci (targeted gene) and novel mutations we identified through linkage analysis and whole genome sequencing. Mutation analysis of known cancer predisposing genes, PTEN, BRCA1, BRCA2, CHEK2, p53, APC, hMLH1, hMSH2, hMSH6, TGFßR1, MYH, ARLTS, DNMT3B, $D N M T 3 A, D N M T 1$ and $C C N D 1$ were performed. In the linkage analysis phase, the whole candidate gene HNRNPAO was sequenced for DNA samples from six cancer carriers and six healthy marry-ins and then the identified novel mutations in this gene were further sequenced for the rest of the family members. For the novel mutation identified WIF1 genes through whole genome sequencing analysis, we performed the mutation analysis on all available family members. 


\section{Linkage analysis}

57 DNA samples including 14 from family members with cancer, 30 from first-degree relatives without cancer and 13 from non-first degree relatives were subjected to linkage analysis. Genotyping was performed on the Illumina Hu-manHap300v2_A chip for 317,498 single nucleotide polymorphic (SNP) markers. We used Merlin [1] and SIMWALK2 [9] programs to perform linkage analysis on this family. Whereas Merlin can effectively deal with markers that are in strong linkage disequilibrium, we had to divide the pedigree into four parts to overcome its limitation on pedigree sizes. We also duplicated a few individuals to assure that we can trace common haplotypes among affected people. We used SIMWALK2 to analyze specific regions of the strongest signals we observed by Merlin program to better delineate the regions of linkage. With SIMWALK2 we analyzed data from the entire family jointly, but we trimmed the markers at a $0.5 \mathrm{cM}$ density to reduce false positive results caused by linkage disequilibrium among the markers. We used a model with a $0.5 \%$ penetrance in non-carriers and $5 \%$ penetrance in carriers. This parametric analysis gives most weight to the affected individuals and becomes essentially model free (since the unaffected do not contribute much to the analysis, and the affected contribute equally to evidence for linkage). Results of multi-point analysis are presented in terms of the LOD score.

\section{Whole genome sequencing (WGS)}

Eight DNA samples including four from cancer affected, one unaffected blood relative, and three healthy marry-ins were subject to whole genome sequencing. Samples were sequenced using the Illumina HiSeq2000 platform with an average depth of coverage of $\sim 47$. The sequences were aligned and the variants were called using the Illumina CASAVA pipelines and re-called using the GATK best practice [2]. Variants called from both pipelines are analyzed but candidate variants that are called from only one of the pipelines were examined against aligned reads from which variants are called. We excluded variants with phredscaled quality score of 20 or less. We also excluded variants from the 1,000 genomes projects to focus on novel variants that exist only in cancer patients in this family. The variants were annotated and analyzed using Variant Tools [8].

\section{Results}

\section{Description of the family}

The family was initially ascertained because of very early onset breast cancer occurring in the proband at age 26 (person 001 in Fig. 1). Subsequently colon cancer arose as $3 \times 3 \mathrm{~cm}$ lobulated rectal adenocarcinoma in an 11-year old girl (individual 144). Subsequent colonoscopies and endoscopies identified $220.2-0.3 \mathrm{~cm}$ sessile polyps throughout the colon, with no evidence of other lesions in the ileum, duodenum, stomach, or esophagus. Upon pathological review, these polyps were all found to have similar morphology being sessile with only minimal loss of crypt orientation. A few crypts were distended by mucin. This child was treated aggressively with local radiation and continuous infusion of $250 \mathrm{mg} / \mathrm{m}^{2}$ per day 5-fluorouracil, a total colectomy with endorectal pull-through and six 5-day courses at 4- to 5-week intervals of $20 \mathrm{mg} / \mathrm{m}^{2}$ intravenous leucovorin and $425 \mathrm{mg} / \mathrm{m}^{2} 5$-fluorouracil. The patient was without disease for a little over 2 years following treatment but eventually 
developed metastases and died at age 16. The pedigree structure, shown in Fig. 1 shows the presence of five prostate cancers (including one occurring at age 47), a melanoma at age 25, and several other cancers, further presented in Table 1.

\section{Targeted mutation analysis}

Germline DNA from individual 001 who had early onset breast cancer was submitted for analysis of PTEN, BRCA1, BRCA2, CHEK2 and $p 53$ analysis, a germline sample from individual 003 was submitted for analysis of APC, hMLH1, hMSH2, hMSH6, TGF $R R 1$, and $M Y H$ and a germline DNA sample from individual 152 was submitted for p16 analysis. No causal mutations were identified in any of these genes. Two sequence variants ofTrp149Stop and Cys148Arg in ARLTS were noted in individual 003. Within the ARLTS gene family members more often carried the Trp149Stop polymorphism than spouses $\left(\chi_{1}^{2}=5.14, p=\right.$ 0.02 ), but these variants were not noted consistently transmitted among family members. Targeted sequencing of $D N M T 3 B, D N M T 3 A, D N M T 1$ and $C C N D 1$ in multiple family members failed to show an association of any polymorphisms in these genes with familial cancer risk.

\section{Linkage analysis}

We performed linkage analysis to identify genetic causes for disease by tracing the coinheritance of genetic markers with disease in families. Results from Merlin showed strongest signals on chromosome 2 (Fig. 2a) and 5 (Fig. 2b). We then used SIMWALK2 for a parametric linkage analysis by using the entire pedigree structure to narrow down the regions of linkage. This analysis supported evidence for linkage to chromosome 5 but denies much evidence for linkage on chromosome 2 giving a maximum location score of 0.562 when 20 markers around the most significant region were studied. Merlin analysis using a parametric model also showed strong evidence for a locus on chromosome 5q (Fig. 2c, d). This locus was inherited much more often than expected by chance with cancer in this family and so suggested that genes in this region influence risk for cancer in this family. We used SIMWALK2 to identify a haplotype that was commonly shared among affected individuals.

Meanwhile, we integrated linkage analysis results with the result from Illumina expression microarray of several family members, in which MOCS2 $(p=0.0026)$ and HNRNPAO $(p=$ 0.0027 ) were found to have the most significant changes in expression levels comparing individuals who have a shared haplotype associated with cancer risk compared with individuals not carrying a risk haplotype, therefore suggesting MOCS2 or HNRNPAO as in the region of linkage between inherited susceptibility to cancers and genetic markers on chromosome 5. No variants were identified in MOCS2 that associated with cancer risk in this family and this gene does not appear to have relevance to cancer susceptibility. The HNRNPAO gene is involved in RNA processing and localizes to the nucleus. Although its role in cancer development is unknown, it belongs to a broader class of proteins governing cell growth. We, therefore, sequenced the entire HNRNPAO gene from six cancer carriers and six healthy marry-in individuals using Sanger sequencing. This sequencing approach identified three novel mutations in this gene [M1: C deletion at position 137091457, M2: G to $\mathrm{C}$ mutation at position 137089865, and M3: $\mathrm{G}$ to A mutation at position 137090925]. M1 
and M2 locate at the $3^{\prime}$ and $5^{\prime}$ UTR of the HNRNPAO gene respectively. Both M1 and M2 locate within the regulatory element of the gene. Predictions from a transcriptional factor search tool (http://www.cbrc.jp/research/db/TFSEARCH.html) suggested that M1 and M2 have an effect on transcriptional factor binding sites, and therefore influence its gene expression.

Next, we sequenced those three mutations on all available family members (including cancer carrier, blood relative and marry-ins) and found that the M1 and M2 mutations were only observed in cancer carriers and blood relative but not in marry-ins. M3 mutations existed in some marrying-in individuals. To further test whether these mutations are family specific, rather than common mutations in cancer patients, we randomly selected 31 DNA samples from patients with Lynch Syndrome to serve as convenient controls for this gene. None of these three mutations were observed for Lynch syndrome patients who are predisposed to development of cancer (data not shown).

Interestingly, we observed one participant (cancer carrier of family member; ID 001) carries a homozygous mutation of M1, which is a deletion at position 137091457, while her mother (sample ID 003) is a heterozygous mutation carrier. We did not have a sample from her father and could not deduce his genotype from the other relatives in the family. To evaluate whether this second hit at this position is a single point mutation or a large fragment deletion, we searched the published SNPs of this gene in NCBI SNPs database. There are a total of 8 SNPs and all of them locate at the $3^{\prime}$ UTR of this gene. We reviewed the sequencing data on the whole gene, but no SNP variations were observed in this particular participant. Failing to observe any variation could reflect either chance homozygosity for all the SNPs or the presence of a deletion on one strand so that the remaining genotyping all appears to result in homozygous genotype results.

Whole genome sequencing analysis-To confirm observed variants on chromosome 5 and uncover other cancer-associated factors which might have been overlooked previously, we sequenced complete genomes of 8 samples, including four cancer carriers, one blood relative, and three marry-ins as controls. The cancer carriers include a patient (sample ID 001) with early onset breast cancer (age 26), her mother (sample ID 003) with breast (age 50) and colon cancer (age 65), an affected relative (sample ID 107) with early onset prostate cancer (age 47) and another affected relative (sample ID 072) with prostate cancer (age 65) who had several offspring affected with cancers. Since we did not have DNA for the girl who died of CRC at age of 16 (sample ID 144), we included her mother (sample ID 134, blood relative), her father (marry-in, sample ID 140), and her grandmother (marry-in, sample ID 132) for whole genome sequencing. In addition, we sequenced another marry-in (sample ID 113). The next generation sequencing data confirmed the M1-M3 mutations observed in HNRNPAO by Sanger sequencing analysis. The result ruled out the possibility of a large deletion on one strand of $H N R N P A O$ in the participant who showed homozygous mutation of M1 and also filtered out the M3 because it was present in some marrying-in individuals.

In summary, including variants called from both the Illumina CASAVA and GATK pipelines but excluding genotypes with quality score lower than 20 , we observed a total of 
$10,669,728$ genetic variants $(8,123,823$ SNPs and 1,970,559 INDELs) from the eight individuals sequenced, with on average 4.77 and 4.47 million variants called by GATK and CASAVA pipelines, respectively. Of these, 68,558 variants exist in all cancer cases and blood relative but are not in the controls. In order to identify novel high-penetrant mutations in this family, we excluded 65,337 variants that were present in the 1,000 genomes project and 53,435 variants from seven controls sequenced for other projects. 2,235 variants remained, among which 28 locate in exon regions of ref seq genes.

We carefully examined these 28 variants against the aligned reads (Table 2). Among those variants, 11 are in mononucleotide or short-tandem repeat sequences and were not consistently called by two pipelines, 4 are in segmental duplication regions with one variant exhibiting extremely high depth of coverage, 2 variants are synonymous and are unlikely to be functional, 1 is observed in the Exome Sequencing project, 5 of them are not in the conserved regions of the human genome, and 2 variants have function estimates but are considered benign by SIFT, PolyPhen2, MutationAssessor, and eXtasy. After removing all these variants, only three variants (HNRNPAO, WIF1 and NPLOC4) left on the list. Although Nuclear protein localization protein 4 homolog (NPLOC4, NPL4) is recognized as a critical component of the endoplasmic reticulum-associated degradation (ERAD) pathway $(1,2)$ and is suspected to be cancer related.

Since the variant in NPLOC4 locates at the $3^{\prime}$ UTR, we used microRNA prediction tool (available at http://www.targetscan.org) to analyze whether this variant falls into a miRNA target site, which might influence expression of the gene. However the prediction shows that this variant doesn't fall into any miRNA target site suggesting that it does not impact microRNA binding. Therefore, we excluded NPLOC4 from further analysis.

The variant on WIFl is reported to be potentially damaging. WIFl is a negative regulator of WNT and hypermethylation of WIFl is commonly observed in many cancers including breast, prostate, bladder, and aerodigestive cancers. This Cys294Phe mutation is predicted to have a strong impact on the structure of the protein but that this position is not evolutionarily conserved. Therefore, we carried out the Sanger sequencing to test Cys294Phe mutation in WIF 1 on every family members. Again these two mutations were only observed in either cancer-carrier or blood-relatives but not in the marry-ins.

\section{The association of the mutation in HNRNPAO and WIF1 and cancer risk}

Next, we performed survival analysis evaluate the association between the mutations we identified in HNRNPAO M2 and WIFl with the cancer risk in this family, either individually or jointly. Both the M2 mutation in HNRNPAO and mutation in WIF1 are significant in both single-variant log-rank test ( $p$ values 0.0001 and 0.0113 , respectively) and cox regression test ( $p$ values 0.0004 and 0.0062 ). When analyzing these two variants jointly, the M2 mutation becomes less significant (with $p$ value 0.0874 ), but effect of WIFI and the interaction between M2 and WIF1 are significant (<0.0001) (Table 3). The Kaplan-Meier survival curves (Fig. 3) show significant differences between family members with and without these mutations individually and jointly, with $p$ values 0.0001 (Fig. 3a), and 0.0066 (Fig. 3b), and 0.0012 (Fig. 3c). All these analyses suggest that the presence of these two 
novel mutations in $H N R N P A O$ and WIF1 are strongly associated with the elevated cancer risk in this family.

The association of HNRNPAO and WIF 1 mutations with gene expression

Next we analyzed the expression data categorized by sample genotype of HNRNPAO mutation 2 and WIFI identified by Sanger sequencing. There were 1,640 genes whose expression levels varied significantly $(p<0.05)$ according to WIF 1 genotypes and 4,303 genes with significant variation by genotype for $\operatorname{HNRNPAO}(p<0.05)$ (Supplemental Table 1). To characterize the effects that these variants have on pathways that may influence cancer risk, we used Ingenuity pathway analysis to organize the gene relationships. As showed in Table 4, the top canonical pathways for HNRNPAO variant were PI3K/AKT signaling $\left(p=6.19 \times 10^{-12}\right)$, ERK/MAPK signaling $\left(\mathrm{p}=9.0 \times 10^{-11}\right)$, NRF2 mediated oxidative stress response $\left(p=6.11 \times 10^{-10}\right)$, B cell receptor signaling $\left(p=1.71 \times 10^{-9},\right)$ and ceramide signaling $\left(p=3.0 \times 10^{-9}\right)$. As showed in Table 5, the top canonical pathways for WIF1 variant were much less significant, including NAD biosynthesis III $\left(p=3.68 \times 10^{-3}\right)$, hepatic fibrosis/hepatic stellate cell activation $\left(p=1.78 \times 10^{-2}\right)$, Proline degradation $(p=$ $\left.2.07 \times 10^{-2}\right)$, nucleotide excision repair pathway $\left(p=2.80 \times 10^{-2}\right)$ and retinoic biosynthesis I $\left(p=3.07 \times 10^{-2}\right)$. Despite the name, the WIF 1 variant did not influence any of the WNT expression levels that are thought to be regulated by WIF1, suggesting that the particular variant of WIF1 in this family did not affect the WIF1 inhibitory function of WNT. The WIF1 protein contains an N-terminal WIF domain and five EGF-like domains. We used NCBI domain search tool and found out that Cys294Phe locates at one of the EGF-like domain other than N-terminal WIF domain. This predication supports our observation of gene expression analysis and pathway analysis.

\section{Discussion}

The present work links the two novel mutations in the HNRNPAO and WIFI genes to the excess cancers in this family. Gene expression study of blood samples showed that the HNRNPAO and WIF1 mutations influence specific cellular signaling pathways related to cancer growth. In addition, we observed a striking interaction between rare variants in HNRNPAO and WIF1 jointly affect cancer risk in an extended pedigree.

HNRNPA0 is a member of the hnRNP A/B family of related RNA binding proteins that bind pre-mRNA and are involved in the processing, metabolism, and transport of nuclear pre-mRNA transcripts [7]. HNRNPA0 is phosphorylated at Ser84 by MAPKAPK-2 in response to LPS treatment in mouse macrophage cells, which might play a key role in stimulating translation of the TNF-pre-mRNA tr [7]. The novel mutation M2 we identified locates at the $5^{\prime}$ UTR of the gene and is predicted to have an effect on transcriptional factor binding sites and therefore influence its expression. On the other hand, WIF1 is a secreted protein that binds to Wnt proteins and inhibits their activity [3]. It contains an $\mathrm{N}$-terminal WIF domain and five EGF-like repeats [4]. It has been reported that WIF1 expression is downregulated in many types of cancers $[5,6,10,11]$.

The region on chromosome 5 with a significant linkage signal is relatively wide and encompasses over 120 genes. Besides three mutations in HNRNPAO, we also identified a 
variant in SQSTM1 on chromosome 5 but outside the linkage region, which demonstrates a significant association with cancer risk in this family ( $p$ value 0.0005), but further sequencing analyses showed that this variant was present in some controls individuals and it was therefore removed from further analyses. Whole genome sequencing also identified a rare mutation in WIF1 that is associated with cancer risk in this family, though not as strongly as the HNRNPAO variant. Joint analysis of WIF1 and HNRNPAO showed a highly significant interaction among carriers of both mutations, but the comparison of the log likelihoods for models with or without the interaction showed only a very modest improvement in the fit of the model with WIFI and the interaction ( $\chi_{2}^{2}=1.04, p=0.57$ ), suggesting that the $H N R N P A O$ variant may be sufficient to explain increased risk for cancer in this family. Further analysis to explore the impact that the HNRNPAO versus WIF1 variants have on expression of genes showed that the HNRNPAO variant influenced well known pathways to cancer development such as PI3K/AKT and MAPK signaling, while the relevance of the WIFI variant to cancer pathways was much less clear. Despite the much stronger prior biological evidence that WIF1 variation could explain risk for cancer in this family and annotation that showed an effect of the WIFl variant on gene function, results for HNRNPAO were much more compelling than for WIF1. Results therefore implicate $H N R N P A O$ as playing the dominant role in cancer risk for this family. Further studies to explore the effects of WIF1 or HNRNPAO on cancer risk will be of value in this family. Given striking interactions that we observed for risk among individuals carrying both variants, further study of this combination could present an opportunity for predictive assessment of risk, but the results are not yet sufficient for clinical action because they have not been validated in a CLIA laboratory and have only been detected in this single family. During the course of the study of this family, two additional cancers arose, both of which carried the rare variants of $H N R N P A O$ and WIFI further strengthening the case for these variations influencing risk. Variations in HNRNPAO might also further be studied for relevance to early onset cancers.

\section{Supplementary Material}

Refer to Web version on PubMed Central for supplementary material.

\section{Acknowledgments}

This study was supported by funding from a private Donor Foundation and Grant CA016672.

\section{References}

1. Abecasis GR, Cherny SS, Cookson WO, Cardon LR. Merlin-rapid analysis of dense genetic maps using sparse gene flow trees. Nat Genet. 2002; 30(1):97-101. [PubMed: 11731797]

2. DePristo MA, Banks E, Poplin R, Garimella KV, Maguire JR, Hartl C, Philippakis AA, del Angel G, Rivas MA, Hanna M, et al. A framework for variation discovery and genotyping using nextgeneration DNA sequencing data. Nat Genet. 2011; 43(5):491-498. [PubMed: 21478889]

3. Hsieh JC, Kodjabachian L, Rebbert ML, Rattner A, Smallwood PM, Samos CH, Nusse R, Dawid IB, Nathans J. A new secreted protein that binds to Wnt proteins and inhibits their activities. Nature. 1999; 398(6726):431-436. [PubMed: 10201374]

4. Liepinsh E, Banyai L, Patthy L, Otting G. NMR structure of the WIF domain of the human Wntinhibitory factor-1. J Mol Biol. 2006; 357(3):942-950. [PubMed: 16476441] 
5. Mazieres J, He B, You L, Xu Z, Jablons DM. Wnt signaling in lung cancer. Cancer Lett. 2005; 222(1):1-10. [PubMed: 15837535]

6. Mazieres J, He B, You L, Xu Z, Lee AY, Mikami I, Reguart N, Rosell R, McCormick F, Jablons DM. Wnt inhibitory factor- 1 is silenced by promoter hypermethylation in human lung cancer. Cancer Res. 2004; 64(14):4717-4720. [PubMed: 15256437]

7. Myer VE, Steitz JA. Isolation and characterization of a novel, low abundance hnRNP protein: A0. RNA. 1995; 1(2):171-182. [PubMed: 7585247]

8. San Lucas FA, Wang G, Scheet P, Peng B. Integrated annotation and analysis of genetic variants from next-generation sequencing studies with variant tools. Bioinformatics. 2012; 28(3):421-422. [PubMed: 22138362]

9. Sobel E, Papp JC, Lange K. Detection and integration of genotyping errors in statistical genetics. Am J Hum Genet. 2002; 70(2):496-508. [PubMed: 11791215]

10. Taniguchi H, Yamamoto H, Hirata T, Miyamoto N, Oki M, Nosho K, Adachi Y, Endo T, Imai K, Shinomura Y. Frequent epigenetic inactivation of Wnt inhibitory factor-1 in human gastrointestinal cancers. Oncogene. 2005; 24(53):7946-7952. [PubMed: 16007117]

11. Wissmann C, Wild PJ, Kaiser S, Roepcke S, Stoehr R, Woenckhaus M, Kristiansen G, Hsieh JC, Hofstaedter F, Hartmann A, et al. WIF1, a component of the Wnt pathway, is down-regulated in prostate, breast, lung, and bladder cancer. J Pathol. 2003; 201(2):204-212. [PubMed: 14517837] 


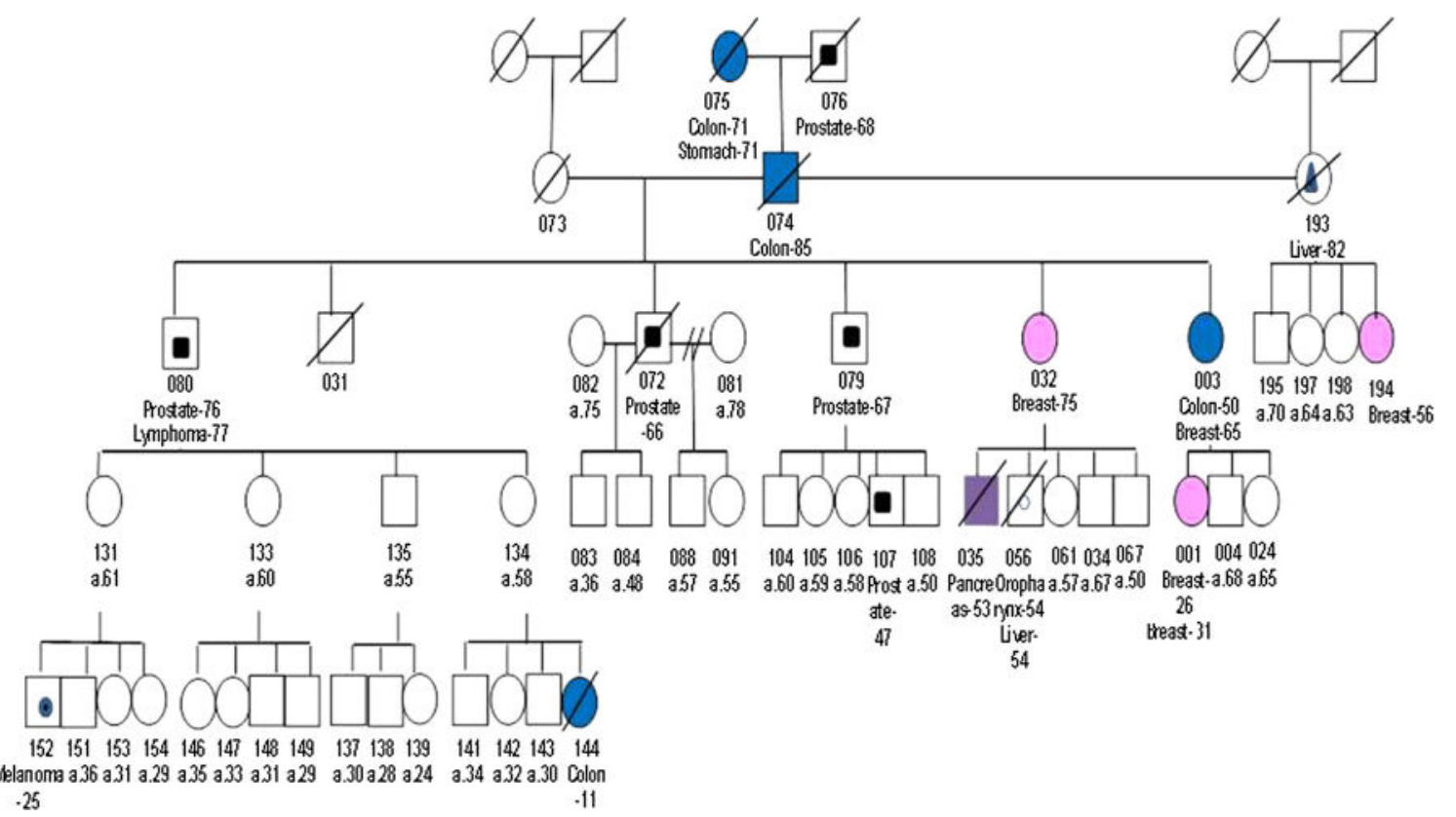

Fig. 1.

Pedigree of the high-risk family 

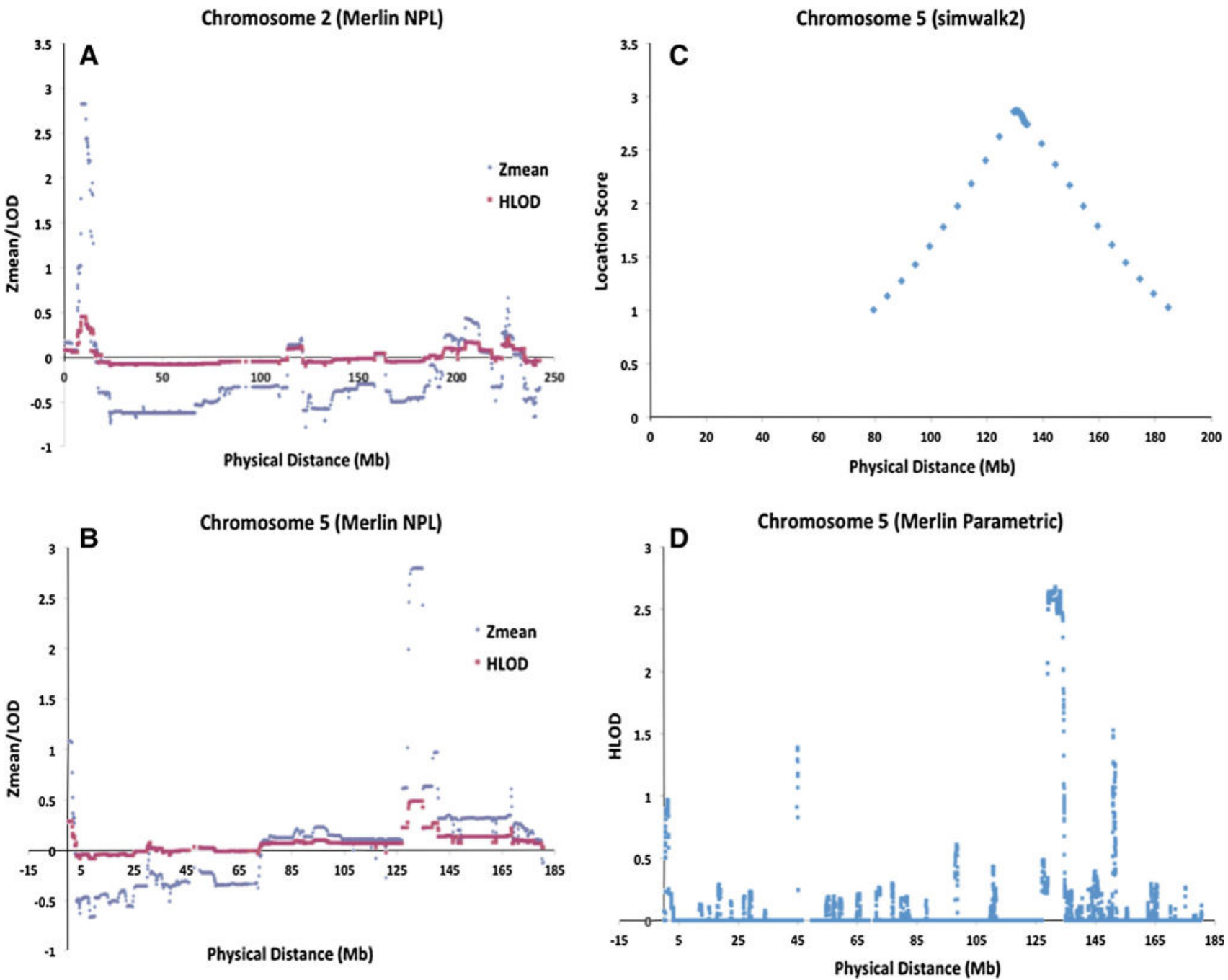

Fig. 2.

Linkage analysis of the family. a, b LOD and Zmean scores on chromosomes 2 (a) and chromosomes 5 (b) using a non-parametric model estimated by Merlin. $\mathbf{c}$ Parametric linkage analysis using SIMWALK2. d Parametric analysis using Merlin 

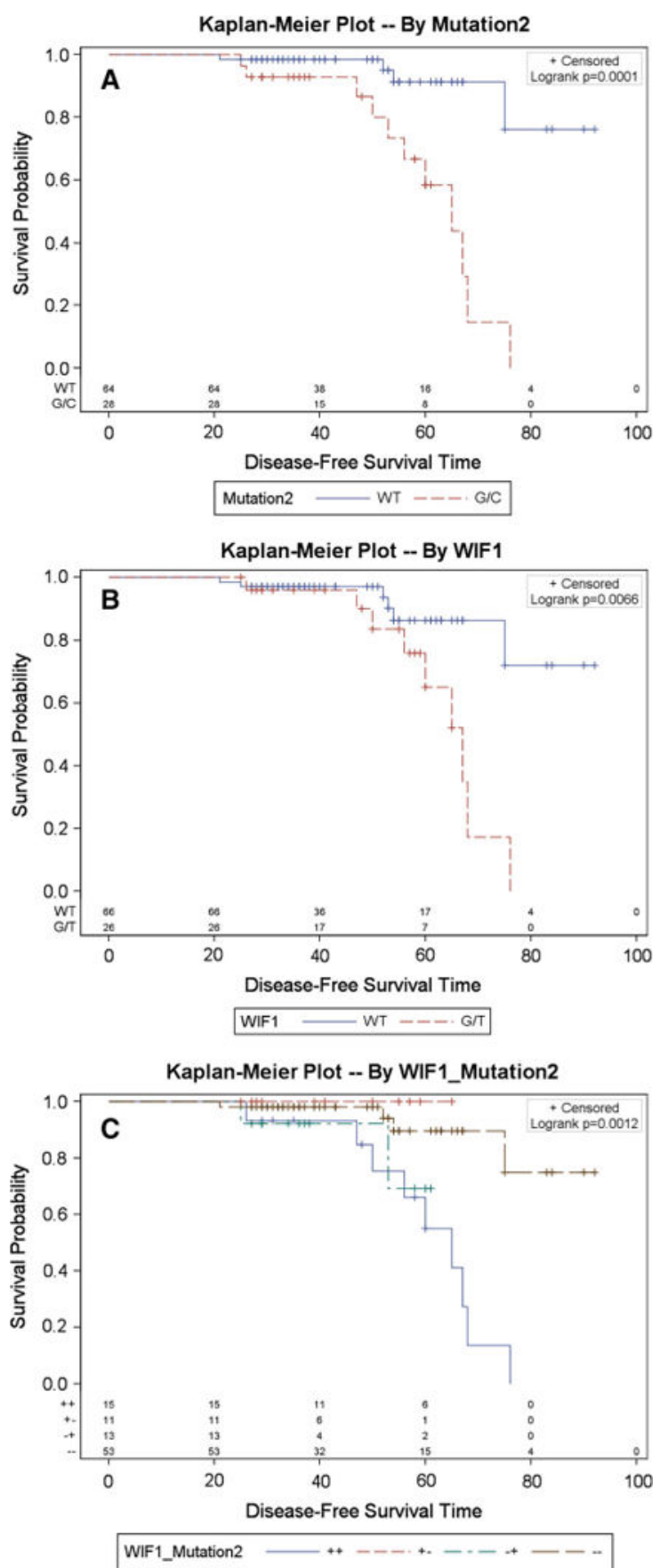

Fig. 3.

Kaplan-Meier survival of family members with and without HNRNPAO and WIF1 mutations. a With HNRNPAO mutations only, b with WIF1 mutations only, $\mathbf{c}$ with both HNRNPAO and WIF1 mutations 


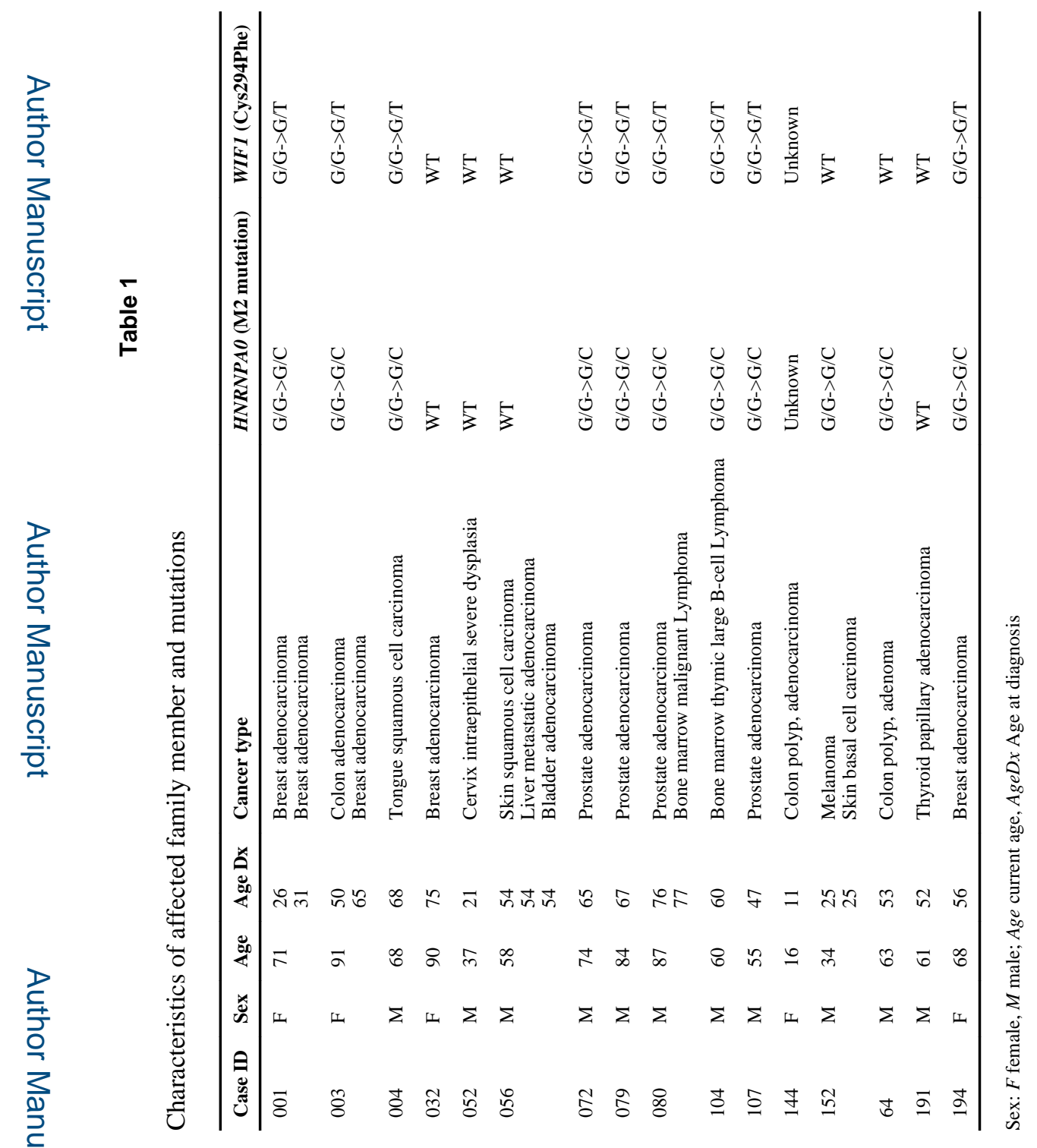

Fam Cancer. Author manuscript; available in PMC 2016 June 01. 


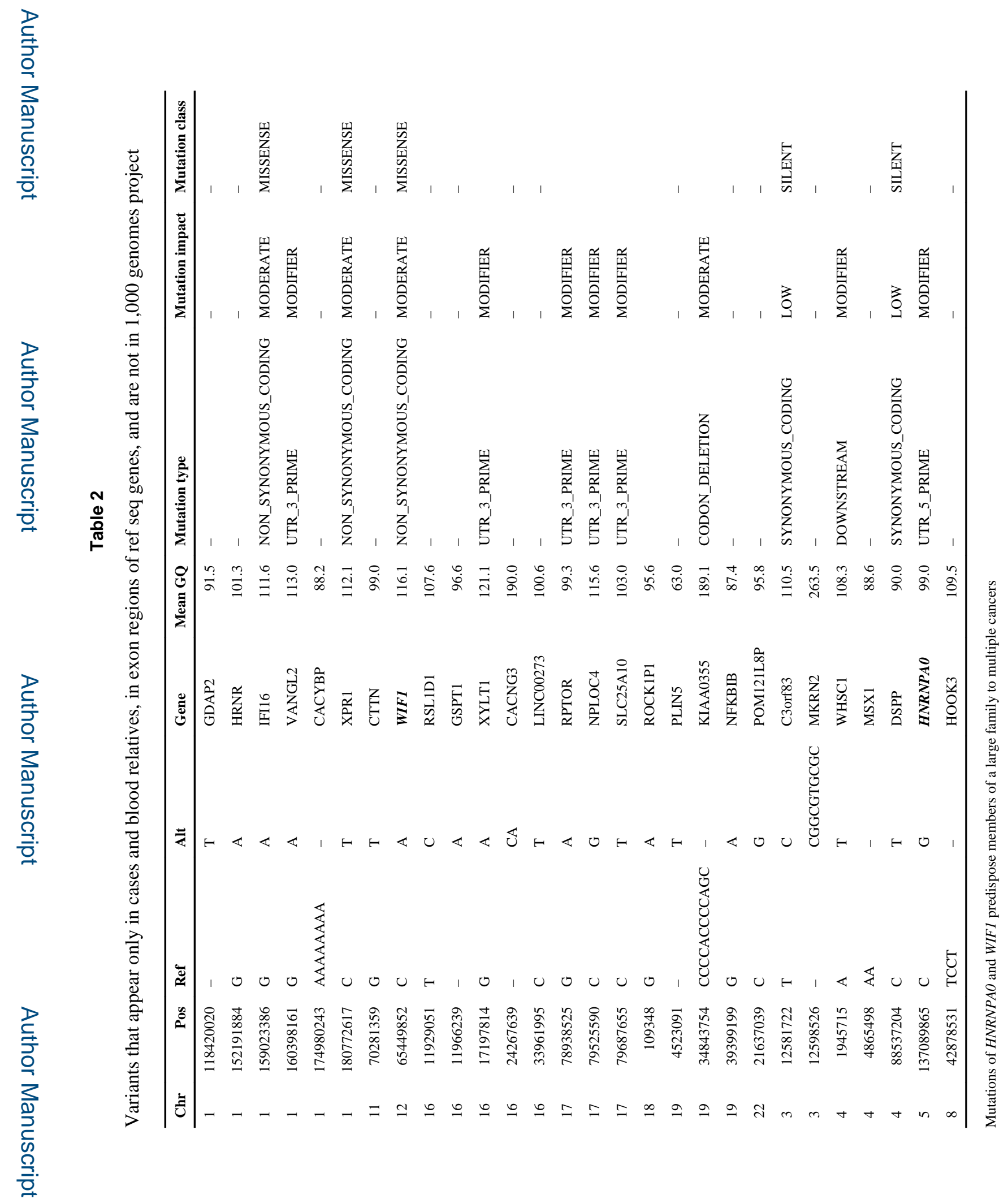

Fam Cancer. Author manuscript; available in PMC 2016 June 01. 


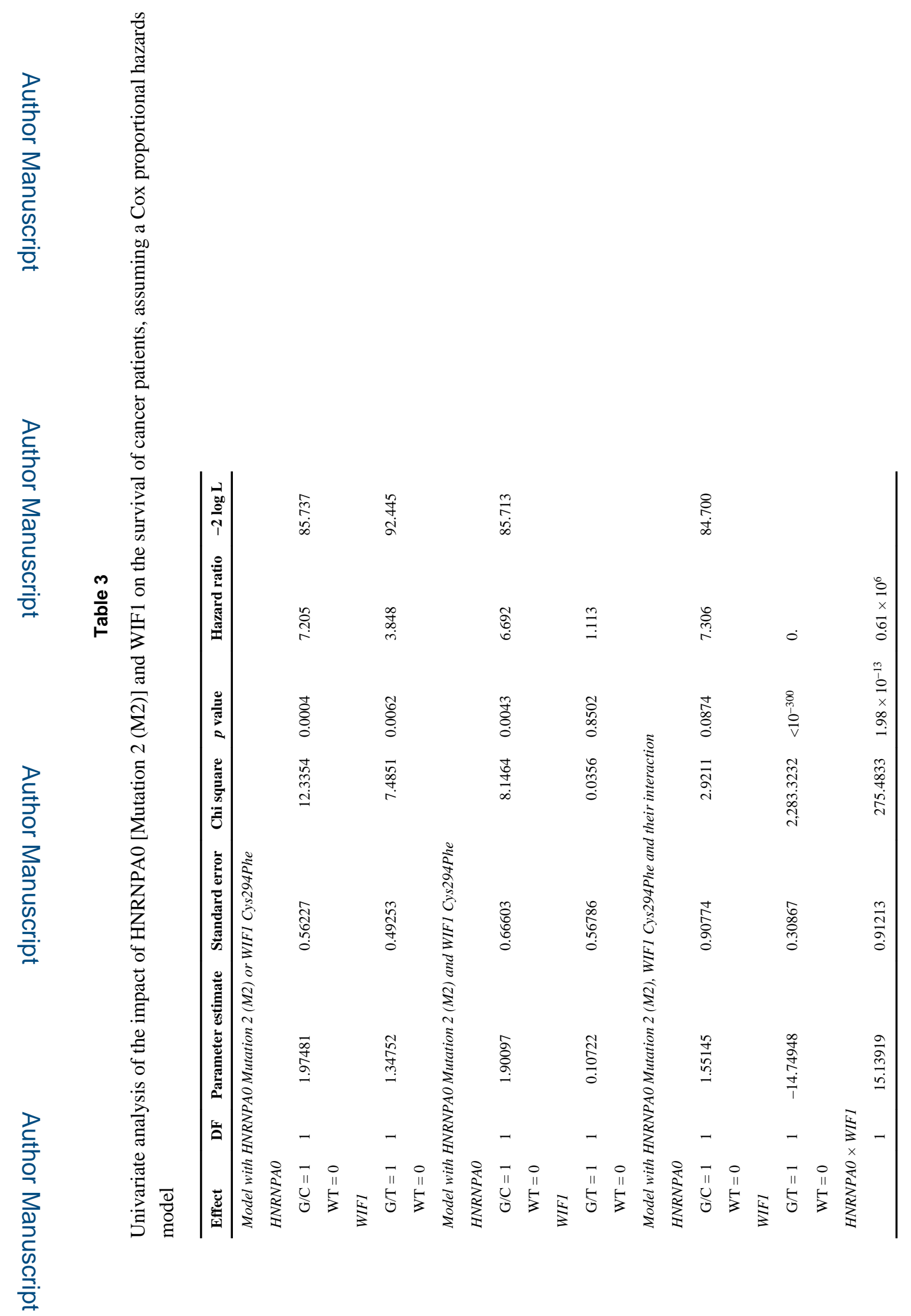

Fam Cancer. Author manuscript; available in PMC 2016 June 01. 


\section{Table 4}

Top canonical pathways of HNRNPA0

\begin{tabular}{lrl}
\hline Name & \multicolumn{1}{l}{ v value } & \multicolumn{1}{l}{ Ratio of affected genes to pathway members } \\
\hline PI3K/AKT signaling & $6.19 \times 10^{-12}$ & \multicolumn{1}{l}{$45 / 152(0.296)$} \\
ERK/MAPK signaling & $9 \times 10^{-11}$ & $56 / 211(0.265)$ \\
NRF2-mediated oxidative stress response & $6.11 \times 10^{-10}$ & $52 / 195(0.267)$ \\
B cell receptor signaling & $1.71 \times 10^{-09}$ & \multicolumn{1}{l}{$48 / 175(0.274)$} \\
Ceramide signaling & $3 \times 10^{-09}$ & $31 / 91(0.341)$ \\
\hline
\end{tabular}


Table 5

Top canonical pathways of WIF1

\begin{tabular}{lcc}
\hline Name & $\boldsymbol{p}$ value & Ratio of affected genes to pathway members \\
\hline NAD biosynthesis III & $3.68 \times 10^{-03}$ & $3 / 10(0.3)$ \\
Hepatic fibrosis/hepatic stellate cell Activation & $1.78 \times 10^{-02}$ & $10 / 155(0.065)$ \\
Proline degradation & $2.07 \times 10^{-02}$ & $2 / 7(0.286)$ \\
Nucleotide excision repair pathway & $2.8 \times 10^{-02}$ & $4 / 36(0.111)$ \\
Retinoate biosynthesis & $3.07 \times 10^{-02}$ & $4 / 37(0.108)$ \\
\hline
\end{tabular}

